\title{
Interfacial Effects on the Dispersion and Dissipation of Shock Waves in Ni/Al Multilayer Composites
}

\author{
Paul E. Specht ${ }^{1,2} \cdot$ Timothy P. Weihs $^{3} \cdot$ Naresh N. Thadhani $^{2}$
}

Received: 29 August 2016/ Accepted: 20 October 2016/Published online: 27 October 2016

(C) Society for Experimental Mechanics, Inc 2016

\begin{abstract}
The influence of interfacial density, structure, and strength in addition to material strengths on the dispersion and dissipation of a shock wave traveling parallel to the layers in a laminar, multilayer composite was investigated using twodimensional, meso-scale simulations incorporating a real, heterogeneous microstructure. Optimum interfacial densities for maximizing wave dispersion and dissipation were identified. Interfacial structure strongly influenced the dispersion by altering the wave interactions internal to the composite. Interfacial strength effected both the dispersion and dissipation through drastic changes to the interfacial strain generated. Lastly, material strength influenced only the dissipation of the shock wave by altering the compressibility of the constituents. The combined results identified interfacial strain as the driving mechanism influencing the shock compression response of the $\mathrm{Ni} / \mathrm{Al}$ multilayered composites.
\end{abstract}

Keywords Composites · Modeling and simulation · Shock loading $\cdot$ Wave propagation

\section{Introduction}

The properties of multilayer composites composed of materials with large differences in their elastic and plastic properties are dominated by their interfaces. In

Paul E. Specht

pespech@sandia.gov

Sandia National Laboratories, Albuquerque, NM, USA

2 Department of Material Science and Engineering, Georgia Institute of Technology, Atlanta, GA, USA

3 Department of Material Science and Engineering, The Johns Hopkins University, Baltimore, MD, USA multilayered materials with nanometer sized layers, the interfaces strongly influence the plasticity mechanisms, leading to very high flow strengths stable to large strains $[1,2]$. In bulk laminated composites, the impedance difference at the material interfaces causes numerous stress wave reflections and interactions affecting the structure of a propagating wave through geometric dispersion and spatial dissipation [3-5]. Geometric dispersion is the spreading of the wave energy that alters the shape of the stress pulse. Spatial dissipation is the deposition of the wave energy irreversibly into the material.

Past experimental work on the shock compression response of laminated composites focused on systems with the layers oriented perpendicular to the direction of shock wave propagation [3-5]. These experiments showed that laminated composites produce periodic perturbations in the shock wave [3]. The perturbations increased with increasing impedance mismatch between constituents [5] and were the main source of attenuation in the material [4]. In addition, extensive analytical work on multilayer composites has examined shock propagation both perpendicular [6-10] and parallel to the interfaces [11-17]. Numerous molecular dynamics studies have also investigated the response of idealized, nanoscale multilayer composites under shock compression [18-20]. However, using mesoscale simulations, the need to stay with idealized, laminar geometries is eliminated and the influence of irregularities in bulk multilayer composites can be understood.

Previous computational work by the authors on the effect of interfacial orientation on the shock compression response of cold-rolled Ni/Al multilayer composites indicated that two dimensional effects caused increased dispersion and dissipation when the shock front traveled parallel to the material interfaces [21]. The differing compressibilities of each material led to areal changes of 
the layers, while the differing wave speeds generated large strains and elevated temperatures at the interfaces. Building on these results, it was desired to examine the effects of various interfacial parameters on the dispersion and dissipation of a shock wave in this "parallel" configuration. Microstructural parameters controllable through fabrication in $\mathrm{Ni} / \mathrm{Al}$ multilayer composites were varied to understand their influence on the dispersion and dissipation response. These characteristics were separated into two categories: interfacial parameters and material properties. Interfacial parameters included interfacial density, structure, and strength, while the material properties focused on the yield strengths of the constituents.

\section{Ni/Al Multilayer Properties}

The multilayer composite used in this work was fabricated from Ni $201(99.6 \% \mathrm{Ni})$ and $\mathrm{Al} 5052 \mathrm{H} 19(2.5 \% \mathrm{Mg}$ and $0.25 \% \mathrm{Cr}$ ) foils initially 178 and $127 \mu \mathrm{m}$ thick, respectively [22]. Using a strain hardened $\mathrm{Al}$ alloy decreased the strength difference between constituents and provided more uniform layering. The multilayer composite had a 1:1 stoichiometric ratio $(60 \% \mathrm{Al}$ and $40 \% \mathrm{Ni}$ by volume) and underwent three rolling cycles. More details on the rolling process are located elsewhere [22].

An optical micrograph of the longitudinal cross-section of the Ni/Al multilayer is shown in Fig. 1. The bright contrast $\mathrm{Al}$ layers were continuous along the length of the composite, while the darker contrast $\mathrm{Ni}$ layers formed elongated particles as a result of necking during rolling. The multilayer exhibited intimate and continuous particle contacts and very limited void space $(\sim 0.25 \%)$. As a result, the multilayer composite was considered fully dense. The multilayer composite had a density of $\rho_{\text {mult }}=$ $5.300 \pm 0.047 \frac{\mathrm{g}}{\mathrm{cm}^{3}}$ and an average bilayer spacing, $\lambda$, of $28.2 \pm 4.2 \mu \mathrm{m}$. The bilayer spacing is the average distance separating two layers of identical material.

\section{Microstructure Generation and Computational Method}

The longitudinal cross section of the cold-rolled multilayer composites have similar characteristics regardless of the number of rolling cycles endured [23]. Utilizing this fact, the optical micrograph shown in Fig. 1 was used for the generation of microstructures with various bilayer spacings through simple scaling to study the influence of interfacial density on the dispersion and dissipation of a shock wave. For bilayer spacings under 28 microns, the periodicity of the multilayer was used to artificially extend the

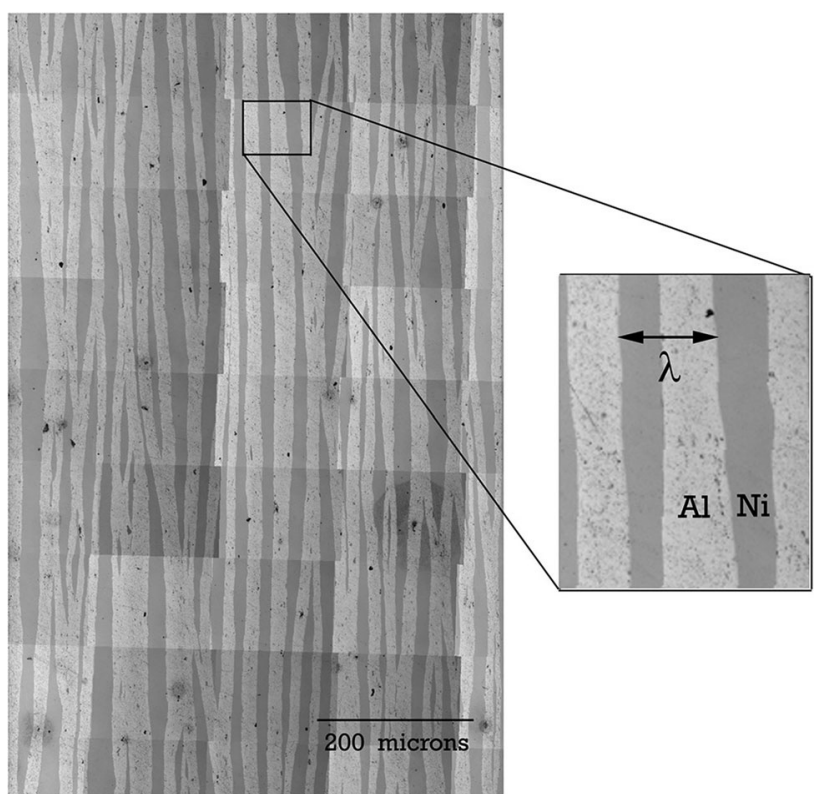

Fig. 1 Optical micrograph of the longitudinal cross-section of the $\mathrm{Ni}$ / Al multilayer composite

microstructure through mirroring. For bilayer spacing larger than 28 microns, the microstructure was taken from a smaller section of the original microstructure and scaled. This procedure enabled the generation of $1 \mathrm{~mm} \times 1 \mathrm{~mm}$ microstructures with average bilayer spacings of $\sim 14,28$, 42, 56 and 112 microns. The CTH renderings for these microstructures are provided in Fig. 2. Each microstructure is referred to by its approximate bilayer spacing (i.e. 14, 28 , 42, 56, and 112 micron configurations).

To provide accurate results, the computational domain must statistically capture the heterogeneities present in the multilayer. A technique for the efficient determination of the representative volume element for a binary, two-dimensional, heterogeneous microstructure is the multi-scale analysis of area fractions (MSAAF), technique developed by Spowart et al. [24]. The MSAAF technique was used to determine that a minimum of nine bilayers were needed to represent the multilayer over a $1 \mathrm{~mm} \times 1 \mathrm{~mm}$ domain to less that $5 \%$ variation in volume fraction. This set the maximum representative bilayer spacing at 112 microns.

The effects of interfacial structure and strength, in addition to material strength, were examined using the 28 micron configuration as a standard. Interfacial structure was investigated by simulating a composite with idealized, uniform layers, termed the "uniform" configuration. To study interfacial strength, the 28 micron composite was simulated with no interfacial strength, termed the "nonbonded" configuration. In both cases, the same material properties as the 28 micron configuration were used. The effect of material strength was investigated by altering the initial yield strengths of each material in the 28 micron 


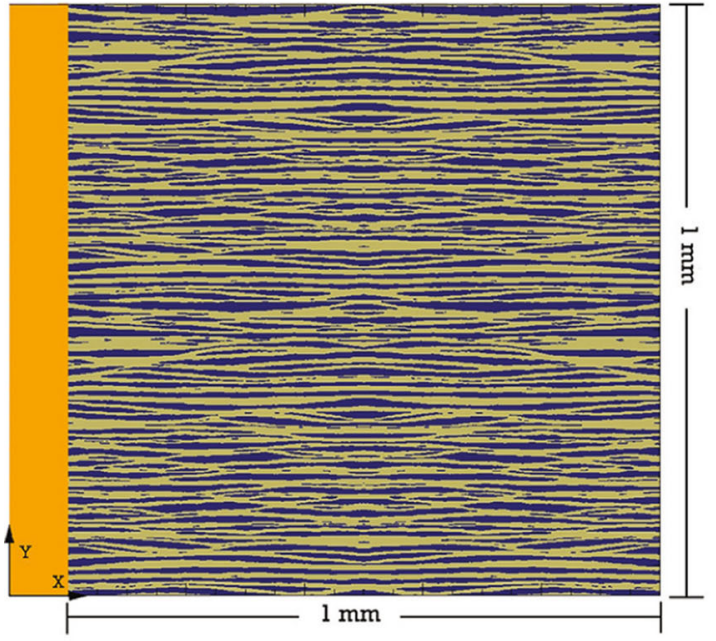

(a)

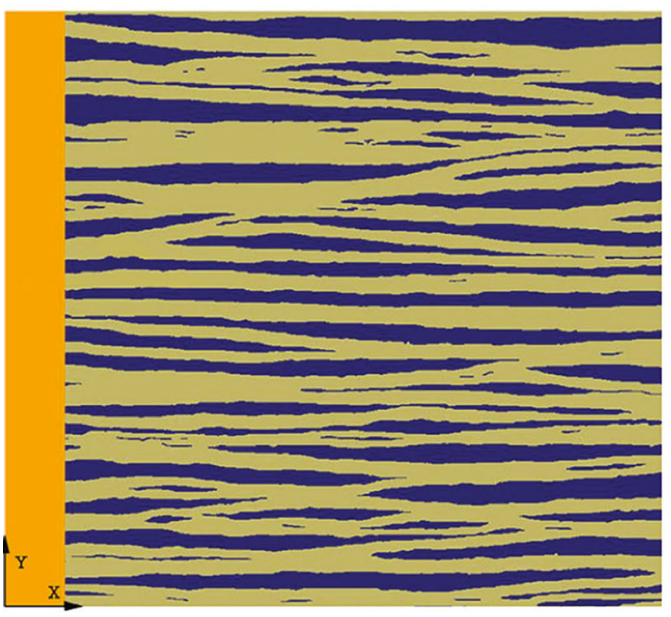

(c)

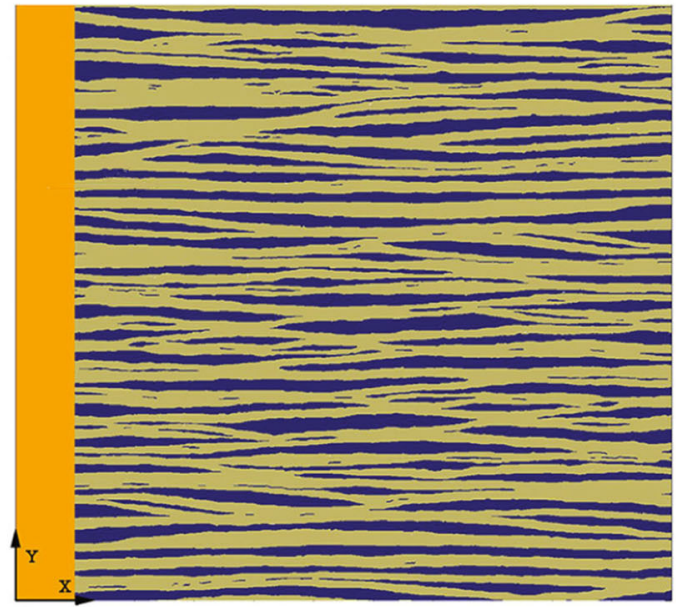

(b)

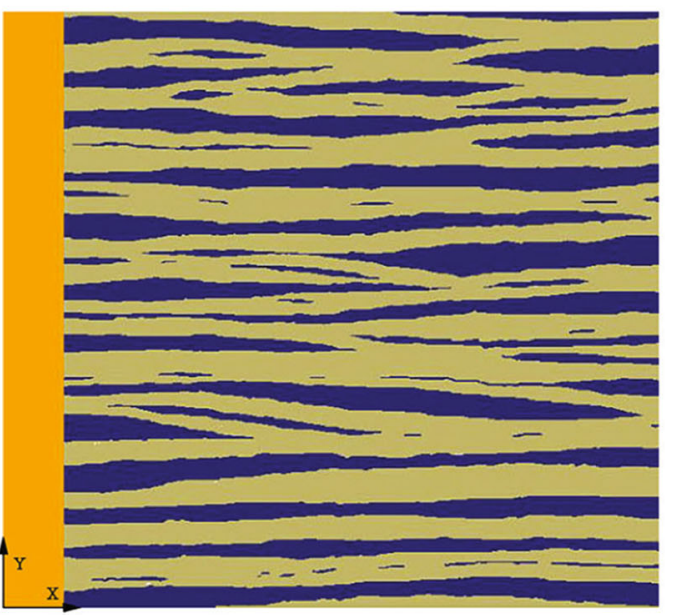

(d)

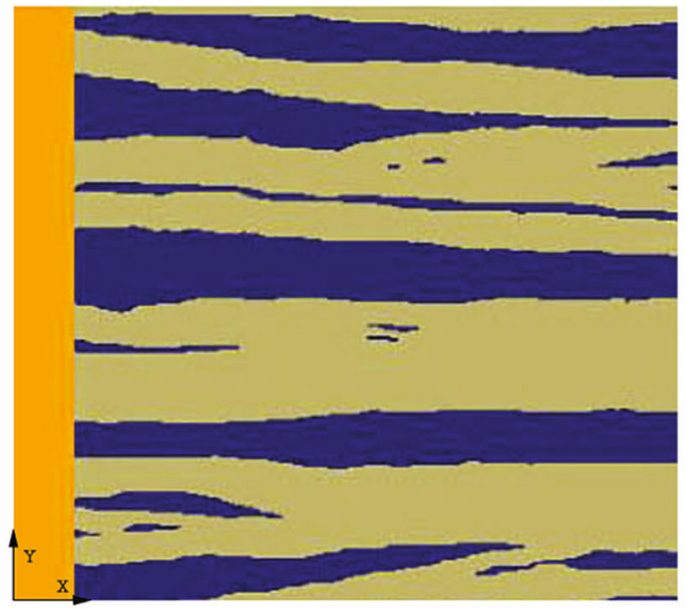

$\mathrm{Al}$
$\mathrm{Ni}$
$\mathrm{Cu}$

(e)

Fig. 2 CTH renderings for multilayer composites with bilayer spacing of a 14, b 28, c 42, d 56, and e 112 microns 
configuration. Two cases were simulated for comparison to the 28 micron configuration: one with strengths of nascent $\mathrm{Ni}$ and $\mathrm{Al}$, termed the "soft" configuration, and one with yield strengths halfway between the nascent materials and the measured values, termed the "half-hard" configuration.

The microstructures shown in Fig. 2 were imported into the multi-material, finite volume, Eulerian hydrocode CTH, developed by Sandia National Laboratories [25], using a MATLAB code [26]. The MATLAB code was developed specifically to incorporate real, heterogeneous microstructures into CTH and captured the heterogeneous nature of the multilayer composites. The computational method employed in this work closely followed that used previously to investigate the effects of interfacial orientation on the shock compression response in identical $\mathrm{Ni} / \mathrm{Al}$ multilayer composites [21]. Uniaxial strain experiments verified that this computational method accurately captured the material response [27].

Impact by a semi-infinite copper piston from the left of the multilayer composite was modeled at five different impact velocities: 500, 750, 1000, 1250, and $1500 \mathrm{~m} / \mathrm{s}$. This yielded particle velocities of around 300, 450, 600, 740 , and $890 \mathrm{~m} / \mathrm{s}$ for each multilayer composite. The multilayer constituents were modeled as $\mathrm{Al} 1100$ and pure $\mathrm{Ni}$, while the piston was modeled as pure $\mathrm{Cu}$. Al 1100 was found to provide an excellent approximation of $\mathrm{Al} 5052$ in both the equation of state (EOS) and constitutive behavior [26]. All materials were modeled using the Mie-Grüneisen EOS. The constitutive behavior of $\mathrm{Cu}$ was represented using the Johnson-Cook model [28] with an infinite yield strength. This ensured the $\mathrm{Cu}$ impactor was rigid and provided a smooth impact surface without any spurious wave reflections. The $\mathrm{Ni}$ and $\mathrm{Al}$ were modeled using the Steinberg-Guinan [29] rate-independent constitutive model. Work hardening of the composites during coldrolling was accounted for by increasing the initial yield strengths of $\mathrm{Ni}$ and $\mathrm{Al}$ to match values obtained from Vickers hardness measurements (Ni 856.45 $\pm 98.07 \mathrm{MPa}$, Al $469.84 \pm 52.30 \mathrm{MPa})$. Stress based fracture was included for both the $\mathrm{Al}$ and $\mathrm{Ni}$ even though no significant tensile stresses were observed. All material interfaces were modeled as perfectly bonded, except for the "non-bonded" configuration. Heat conduction for each material was incorporated through tabular data. Periodic boundary conditions were used in the y-direction, while the boundaries in the $\mathrm{x}$-direction were modeled as sound speed-based absorbing to approximate semi-infinite materials.

Special consideration was given to the computational mesh. Even though the microstructures were scaled versions of each other, the mesh resolution can not scale accordingly. CTH is a scale independent code. If the cell size scales with the microstructure, the simulations are essentially just various domain sizes of the same microstructure. As a result, the cell size was kept constant for each simulation and corresponded to that which provided convergence for the smallest bilayer spacing. Convergence was found at a resolution of approximately 17 cells across each layer [21]. This yielded a resolution of 0.4 $\mu \mathrm{m} / \mathrm{cell}$ for the 14 micron composite. A constant mesh resolution is not the same as having higher resolved meshes for the larger bilayer spacing configurations. While the number of cells increased across the layers, the simulations maintained a similar time step and a consistent thermal length scale. This minimized numerical artifacts so variations in response were directly attributed to the interfacial density.

In order to understand the bulk shock compression response of each multilayer composite, particle velocity $\left(U_{P}\right)$ and wave front velocity $\left(U_{W}\right)$ relationships were determined. The particle velocity was calculated from ten Lagrangian tracer points located in the $\mathrm{Cu}$ driver. To compute the wave front velocity, a MATLAB script was used to obtain an average pressure along the length (at each $\mathrm{x}$ location) of the multilayer composite, referred to as a pressure trace. With a shock wave traveling parallel to the interfaces, extensive two-dimensional effects develop due to the differing wave speeds in each constituent. This generates large amounts of dissipation and dispersion, smearing the shock front as the wave propagates through the composite [21]. As a result, the velocity of the wave front varies strongly with pressure. In order to facilitate comparisons, a wave front velocity, $U_{W}$, corresponding to $25 \%$ of the steady state pressure was defined. This definition was consistent with previous work on multilayer composites [21] and enabled characterization of the bulk parameters of each multilayer composite. Since the bulk parameters can not represent all of the complexities occurring in the shock front, the interfacial responses were further investigated using high resolution adaptive mesh refinement (AMR) simulations. A small section of each composite impacted at $1000 \mathrm{~m} / \mathrm{s}$ was resolved to $100 \mathrm{~nm} /$ cell in order to visualize the changes in temperature and strain at the interface.

\section{Quantification of Bulk Dispersion and Dissipation}

Metrics were developed to characterize the dispersive and dissipative behaviors of each composite. Dispersion in a material is best viewed by looking at the rise of the shock pulse, since it clearly illustrates the distribution of the wave energy. While energy dissipation also effects the rise time, dispersion is the dominant factor. This enabled the 1D pressure traces to quantify the observed bulk dispersion in each simulation. The pressure traces for each configuration 
corresponding to $100 \mu \mathrm{s}$ after impact at $1000 \mathrm{~m} / \mathrm{s}$ were compared. This method provided no insight into the dispersion perpendicular to the propagating shock wave, which was minimal.

The bulk dissipation was determined through the EOS. The area under the Rayleigh line represents the energy deposited into the system upon loading. Since the material unloads along the isentrope, the area under that curve represents the energy recovered. For the pressures investigated, the Hugoniot approximates the isentrope to high accuracy. The energy dissipated was calculated from the area between the Rayleigh line and Hugoniot. While the 1D Rankine-Hugoniot conditions do not fully account for the $2 \mathrm{D}$ effects present in the parallel configuration, the assumption was consistent among all configurations and enabled the extraction of trends from the bulk dissipation results. The bulk dissipation responses were further characterized using the shock rise times and high resolution AMR simulations.

\section{Simulation Results}

Using the same procedure described by Specht et al. [21], a linear EOS $\left(U_{W}=C_{0}+S_{1} U_{P}\right)$ was fit to each microstructural variation. The inert sound speeds, $C_{0}$, and material constants, $S_{1}$, corresponding to the $U_{W}$ versus $U_{P}$ response for each configuration, valid for $U_{P}<1000 \mathrm{~m} / \mathrm{s}$, are given in Table 1. The influence of composite properties are individually addressed in the following subsections.

\section{Effect of Interfacial Density on Shock Wave Dispersion and Dissipation}

The pressure profiles for the various bilayer spacings are shown in Fig. 3a. To aid in comparison and clearly display the trends, the curves were shifted along the abscissa. The

Table $1 U_{W}$ versus $U_{P}$ least squares fits for each multilayer configuration for $U_{P}<1000 \mathrm{~m} / \mathrm{s}$ along with the corresponding $95 \%$ confidence interval.

\begin{tabular}{lll}
\hline Configuration & $C_{0}(\mathrm{~m} / \mathrm{s})$ & $S_{1}$ \\
\hline 14 micron & $4491 \pm 52$ & $1.572 \pm 0.084$ \\
28 micron & $4408 \pm 60$ & $2.003 \pm 0.096$ \\
42 micron & $4533 \pm 163$ & $1.981 \pm 0.258$ \\
56 micron & $4642 \pm 150$ & $1.989 \pm 0.238$ \\
112 micron & $5227 \pm 106$ & $1.492 \pm 0.165$ \\
Soft & $4753 \pm 45$ & $1.600 \pm 0.073$ \\
Half-hard & $4474 \pm 148$ & $1.888 \pm 0.223$ \\
Uniform & $4276 \pm 128$ & $1.969 \pm 0.205$ \\
Non-bonded & $5126 \pm 50$ & $1.482 \pm 0.079$ \\
\hline
\end{tabular}

14 micron configuration had a shorter rise time compared to the 28 micron configuration. The rise time continued to increase as the bilayer spacing increased from the $28 \mathrm{mi}$ cron to the 56 micron configuration. In addition, a dual wave structure emerged, indicative of a leading wave traveling through the lower impedance $\mathrm{Al}$ followed by a slow rise to the final equilibrium pressure. For the 112 micron configuration, the rise time decreased drastically, becoming similar to that of the 14 micron configuration. Simulations performed with a $2 \mathrm{~mm}$ long domain showed no significant change in rise time for all configurations, indicating the reported trends are representative of the steady, equilibrium response. These results suggest that maximum dispersion occurs at a bilayer spacing around 50 microns, and is consequence of the two dimensional nature of the multilayer response.

At high interfacial densities (e.g. the 14 micron configuration) two dimensional effects were less

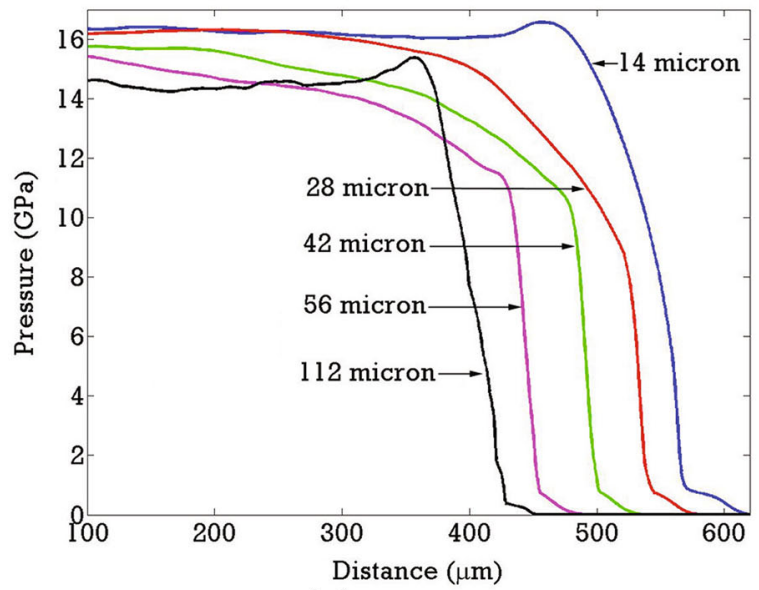

(a) Rise Times

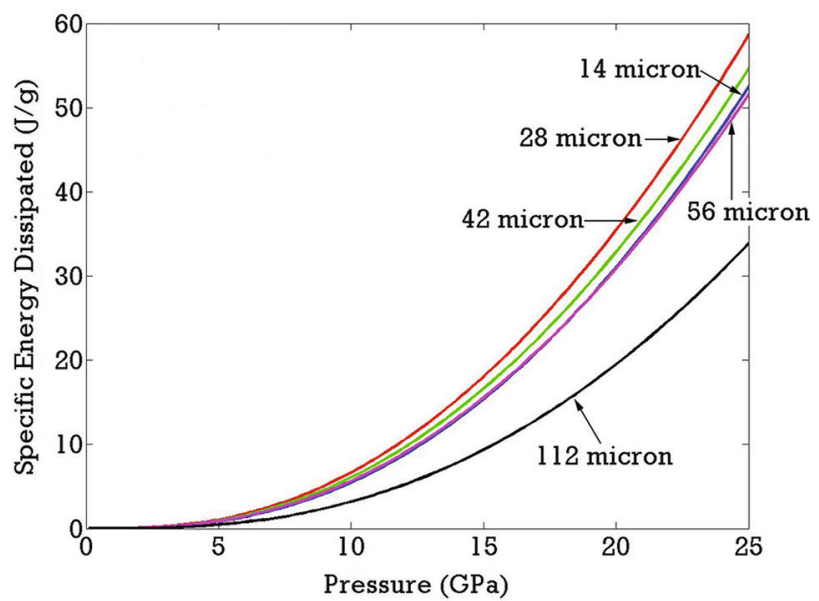

(b) Energy Dissipated

Fig. 3 The effect of bilayer spacing on the a dispersion and b dissipation of the shock front. Both the dispersion and dissipation were maximized over the bilayer spacing range investigated 
pronounced and the smaller separation of materials induced more wave interactions. This equilibrated the system to a singular material velocity quickly, smearing out the shock front. As the interfacial density decreased, the separation of materials increased and fewer internal wave interactions were generated. This enabled a sustained lead wave and generated longer equilibration time to the final pressure. Eventually, the interfacial density decreased enough (e.g. 112 micron configuration) that the interfaces were no longer a dominant mechanism for the dispersion of the wave. There were so few wave interactions, the shock front was not disturbed appreciably from its initial form, reducing the rise time.

Figure $3 \mathrm{~b}$ shows the specific energy dissipated at various shock pressures for each bilayer spacing. While the dissipation increased from the 14 micron to the 28 micron configurations, further increases in bilayer spacing led to a decrease in dissipation. A peak in dissipation occurs for a bilayer spacing of around 30 microns. The mechanisms responsible for this are clearly illustrated with the higher resolution AMR simulations.

The temperature profile generated with the high resolution AMR simulations for the 14 micron configuration is shown in Fig. 4a. A histogram corresponding to this temperature profile is shown in Fig. 5a. Al layers exhibited elevated temperatures compared to the $\mathrm{Ni}$, since it was more compressible. The fast equilibration of the 14 micron configuration not only caused a shorter rise time, but also affected the interfacial temperatures and strains. With fast equilibration of the system, the disparity in material velocities between $\mathrm{Ni}$ and $\mathrm{Al}$ did not persist long. This effect, coupled with the high interfacial density, meant each interface underwent less strain and did not produce highly elevated temperatures (Fig. 4a). This was supported by the temperature histogram shown in Fig. 5a which had one large, broad peak and an insignificant tail.

The dissipation increased in the 28 micron configuration due to increased interfacial strain. The longer equilibration time caused the disparity in material velocities between

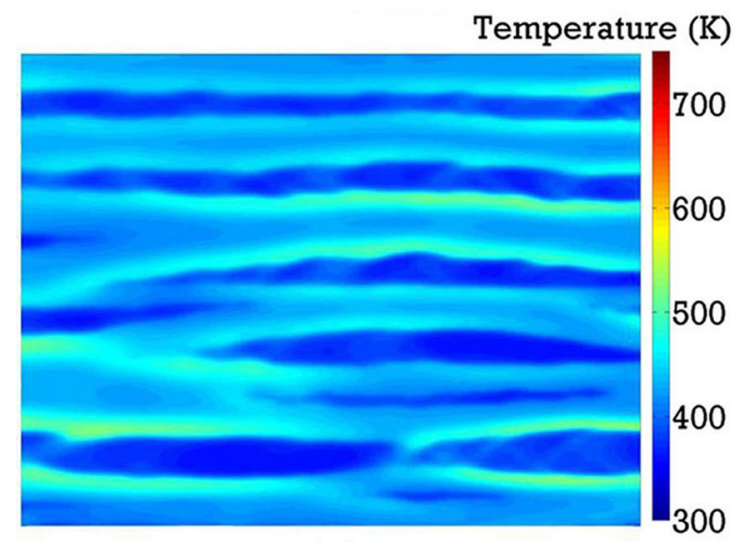

(a)

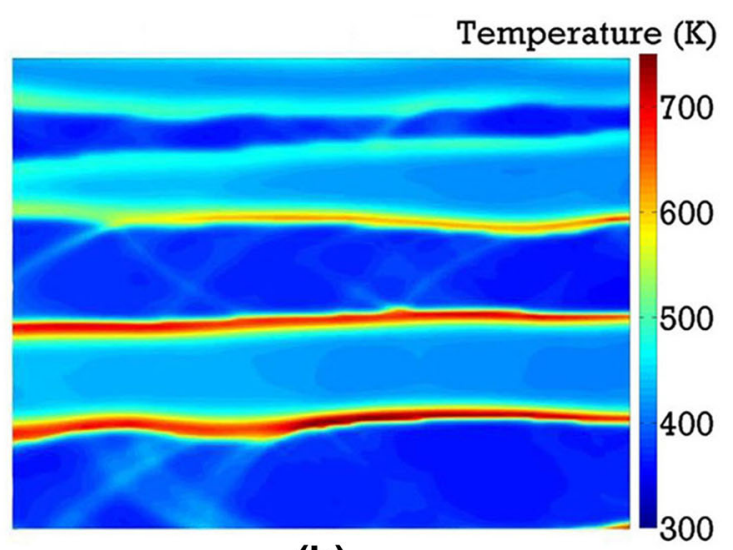

(b)

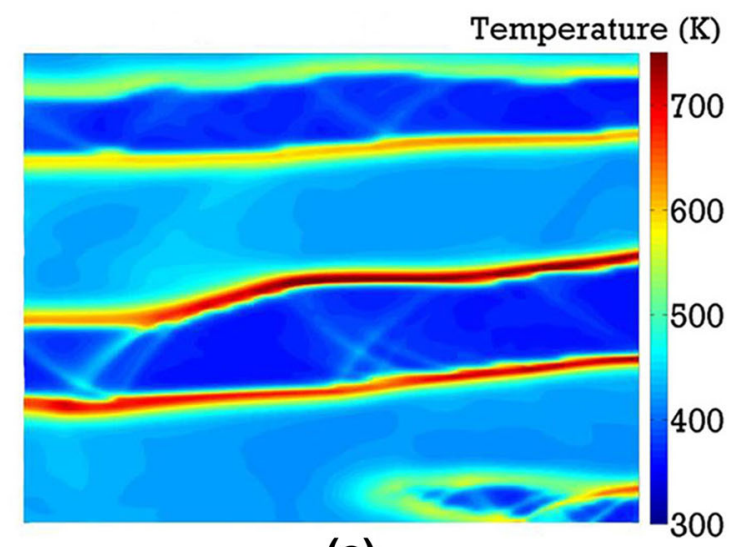

(c)

Fig. 4 High resolution AMR simulations showing the temperature profiles for the a 14, b 28, and c 56 micron bilayer configurations. As the bilayer spacing was increased, the interfacial temperatures increased due to a more prolonged disparity between material velocities 


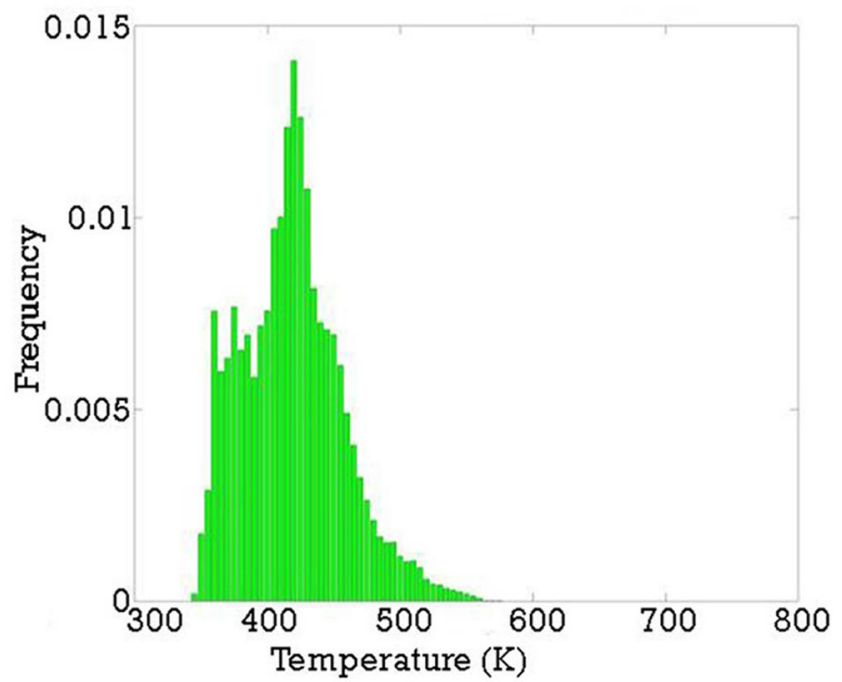

(a)

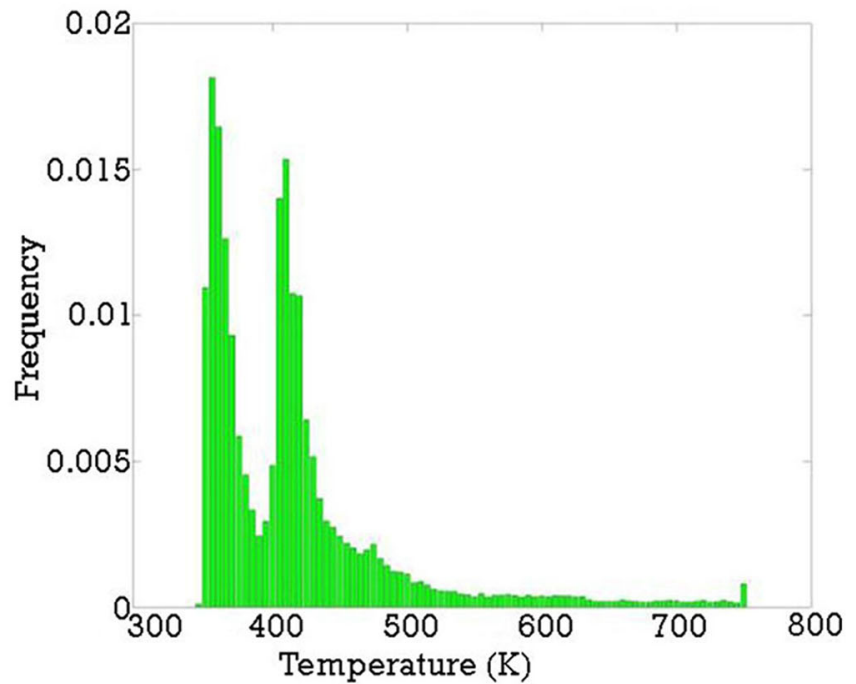

(b)

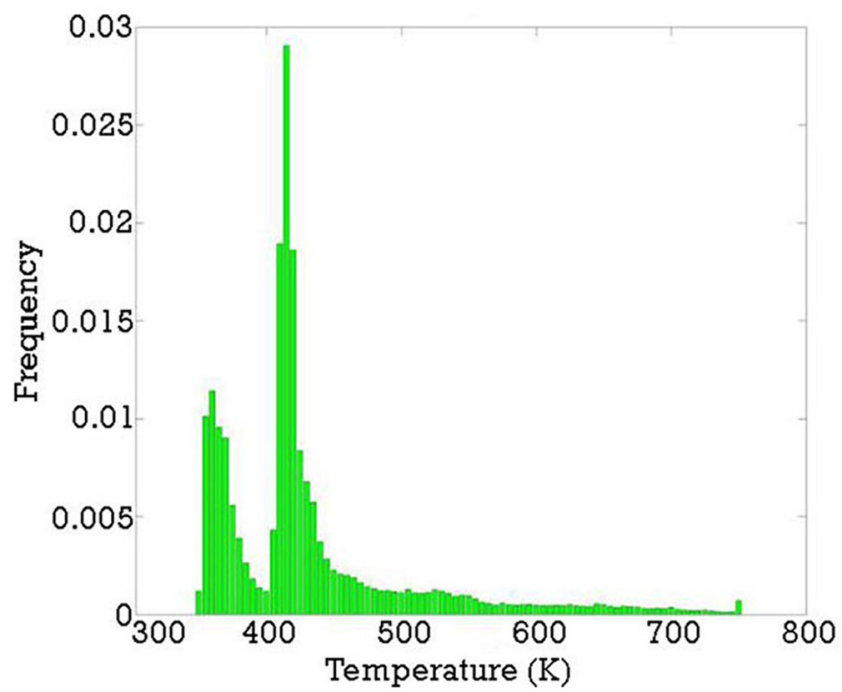

(c)

Fig. 5 Temperature histograms for the high resolution simulations on the a 14, b 28, and c 56 micron bilayer configurations. The 14 micron simulation had a single large, broad peak with no tail, due to the faster equilibration of the composite and the low interfacial temperatures.

constituents to persist longer. This, coupled with the decrease in interfacial area, generated more interfacial strain to accommodate the deformation and produced elevated interfacial temperatures (Fig. 4b). With increased separation of the materials, the individual layers maintained larger temperature differences generating the bimodal histogram seen in Fig. 5b. The first peak represents the cooler Ni layers, while the second represents the warmer Al layers. The long tail of the distribution corresponds to the higher temperatures at the material interfaces.
The histograms for the 28 and 56 micron configurations exhibited two peaks corresponding to the temperatures seen in each material and extensive tails representing the elevated interfacial temperatures

This increased dissipation at each interface was large enough to cause the 28 micron configuration to be more dissipative than the 14 micron configuration despite the loss of interfacial area.

As the bilayer spacing increased further to 56 microns, the dissipation decreased. The further separation of the materials had not dramatically affected the equilibration time compared to the 28 micron configuration. As seen in Fig. 4b, c, similar interfacial strains and temperatures were generated in the 56 micron configuration as in the 28 


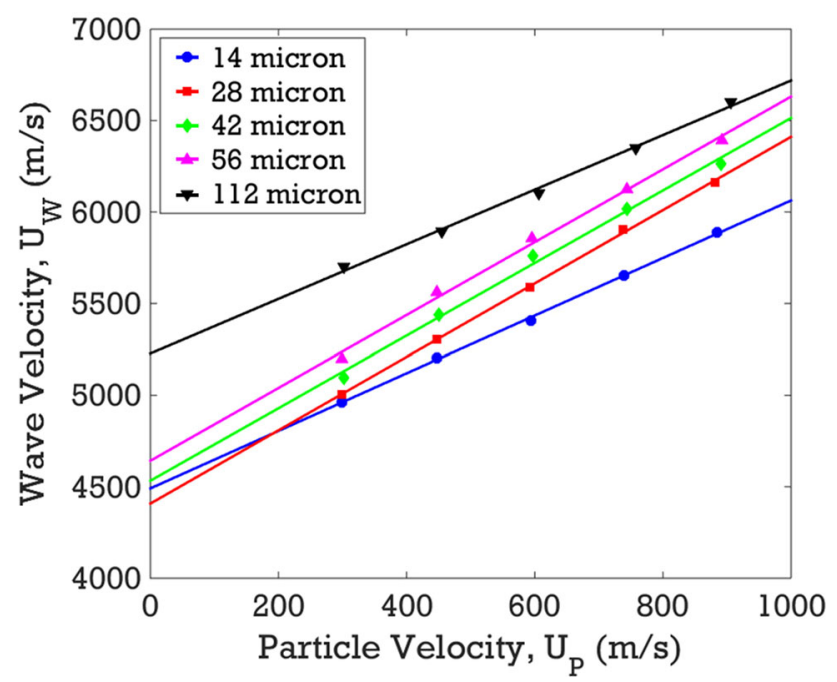

Fig. $6 U_{W}$ versus $U_{P}$ relationship for the different bilayer spacings for $U_{P}<1000 \mathrm{~m} / \mathrm{s}$. The markers correspond to the simulation results, while the lines represent the least squares fits

micron configuration. Additionally, the temperature histogram corresponding to the 56 micron configuration, seen in Fig. 5c, is similar to the histogram for the 28 micron configuration (Fig. 5b). This suggests the interfaces are only supporting a modest increase in strain. This slight increase in dissipation per interface was not sufficient to offset the loss of interfacial area, causing the downturn in dissipation with increasing bilayer spacing after the 28 micron configuration.

The differing dispersive and dissipative characteristics of each configuration altered their EOS responses, which are shown graphically in Fig. 6. It is hard to draw strict comparisons between $C_{0}$ and $S_{1}$ with dispersion or dissipation since both phenomena alter the value of these parameters. However, using the present results, it became apparent that a decrease in dispersion or dissipation produced a shallower slope and increased sound speed in the multilayer composites. This was evident in the similar slopes between the two extreme configurations (14 and 112 micron) and the three middle configurations (28, 42, and 56 micron). An additional observation was seen in the position of each of these curves in the $U_{W}$ versus $U_{P}$ plane. As the bilayer spacing increased, the EOS response shifted upwards to higher shock wave speeds. With a lower interfacial density, there were fewer obstacles to inhibit wave motion and the composite exhibited a lower impedance.

\section{Effect of Interfacial Structure and Strength on Dispersion and Dissipation}

Interfacial structure was investigated by comparing the responses of a cold-rolled multilayer, represented by the

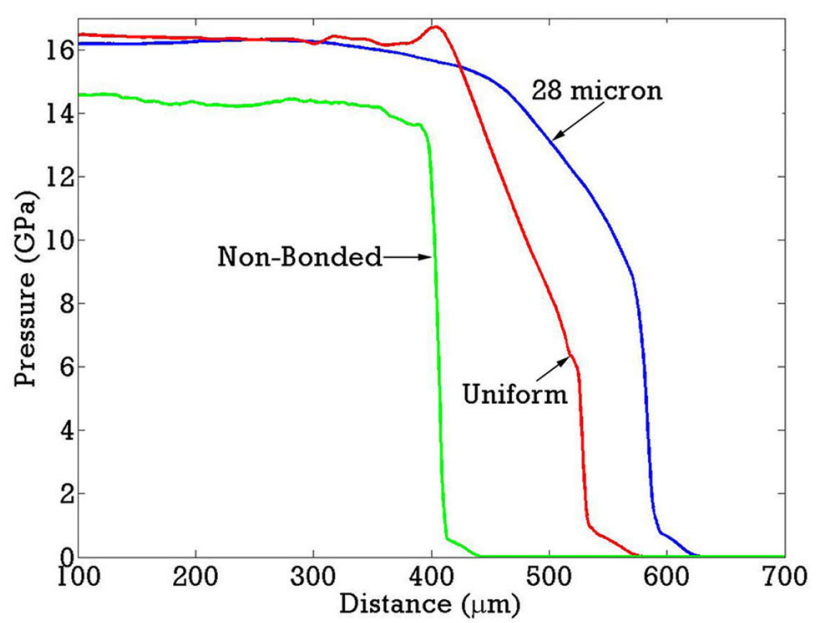

(a) Rise Times

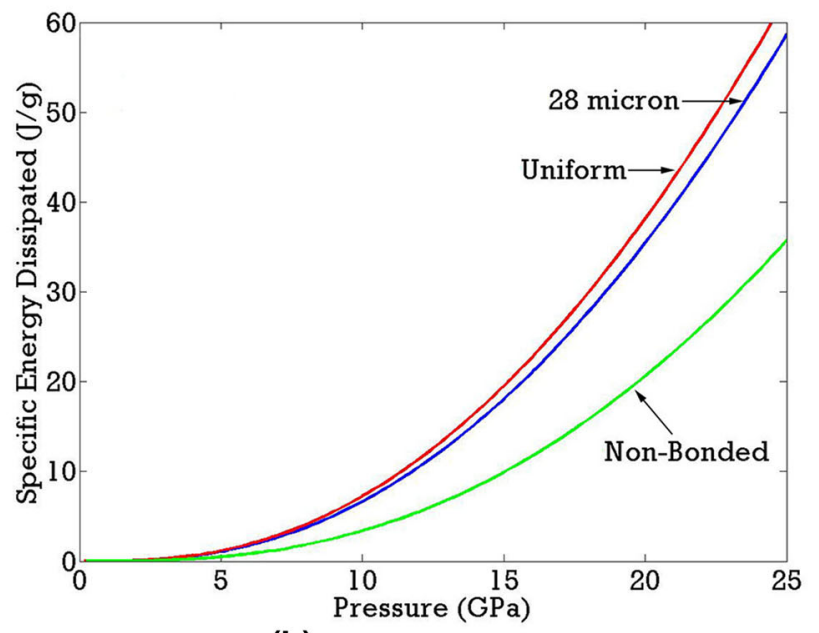

(b) Energy Dissipated

Fig. 7 The effect of interfacial structure and strength on the a dispersion and $\mathbf{b}$ dissipation of the shock front. Heterogeneities generated through rolling only slightly effected dispersion and dissipation, while the interfacial strength had a dramatic effect

28 micron configuration, to that of a uniformly layered composite with the same bilayer spacing, constituent ratio, and material properties. To investigate the effects of interfacial coherency, the 28 micron configuration was simulated with both perfectly bonded and completely unbonded interfaces.

The shock fronts for these three configurations are presented in Fig. 7a. Once again, the curves are shifted along the abscissa to more clearly illustrate the trends. The "uniform" composite had a clear dual wave structure, due to the differing wave speeds of $\mathrm{Ni}$ and $\mathrm{Al}$. The heterogeneities generated during rolling obscured this dual wave structure, smoothing the wave front without altering the rise time. This implied that rolling only slightly increased the dispersion of the wave, and suggests that the dispersion of the wave was influenced more by the density of the 


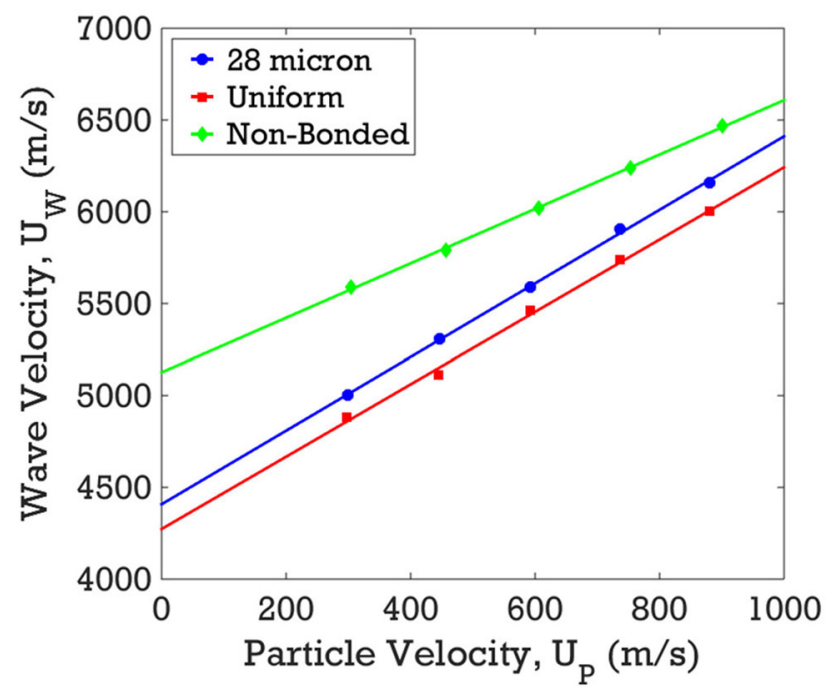

Fig. $8 U_{W}$ versus $U_{P}$ relationship for the "uniform", "non-bonded", and 28 micron composites for $U_{P}<1000 \mathrm{~m} / \mathrm{s}$. The markers correspond to the simulation results, while the lines represent the least squares fits

interfaces than their structure. For the "non-bonded" composite, the wave dispersion decreased dramatically. Without interfacial strength, the materials moved freely. This meant that the shock front in the Al only dissipated energy through compaction of the nascent Ni layers. This was also why the "non-bonded" composite response slowly increased to the equilibrium pressure after the front.

The effects of interfacial strength and structure on the bulk dissipation response are presented in Fig. 7b. Interfacial structure slightly effected the dissipation, with the uniform composite being slightly more dissipative than the cold-rolled composite. With uniform layering, all material interfaces were aligned perfectly with the propagating shock wave. This maximized the interfacial shear generated by the disparity in material velocities between the layers. In contrast, the effect of interfacial strength had a significant effect on the bulk dissipation. With no interfacial strength, no interfacial shear was generated, making compression the only mechanism for energy dissipation. The elimination of the primary mechanism for energy dissipation in the system caused the dramatic drop in dissipation of the "non-bonded" configuration seen in Fig. 7b.

The dispersive and dissipative characteristics are seen in the $U_{W}$ versus $U_{P}$ plots for each composite shown in Fig. 8 . The lower dispersion and dissipation in the "non-bonded" configuration decreased the material slope and increased the inert sound speed compared to the 28 micron composite. This was consistent with the observations made for the various bilayer spacings. The 28 micron and "uniform" composites had similar slopes, but different inert sound speeds. This came from the somewhat off-setting combination of an increase in dissipation and a decrease in

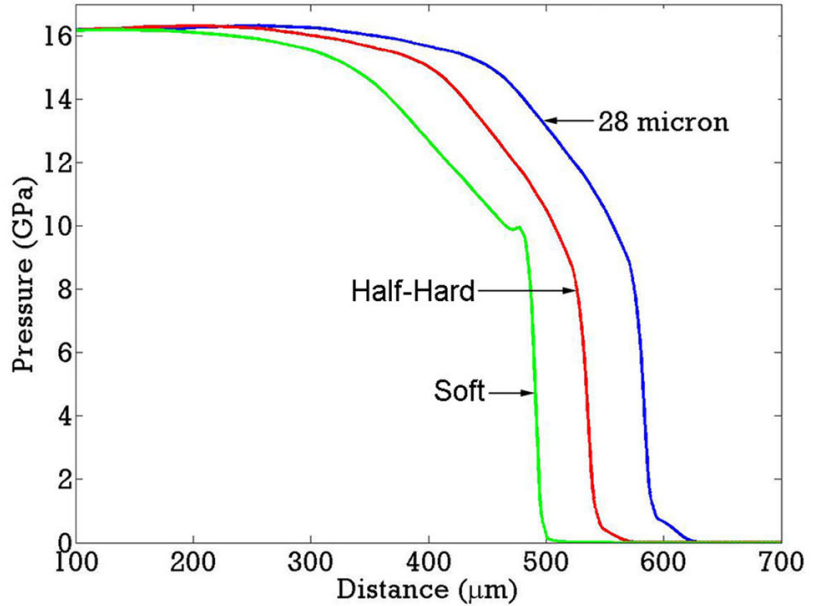

(a) Rise Times

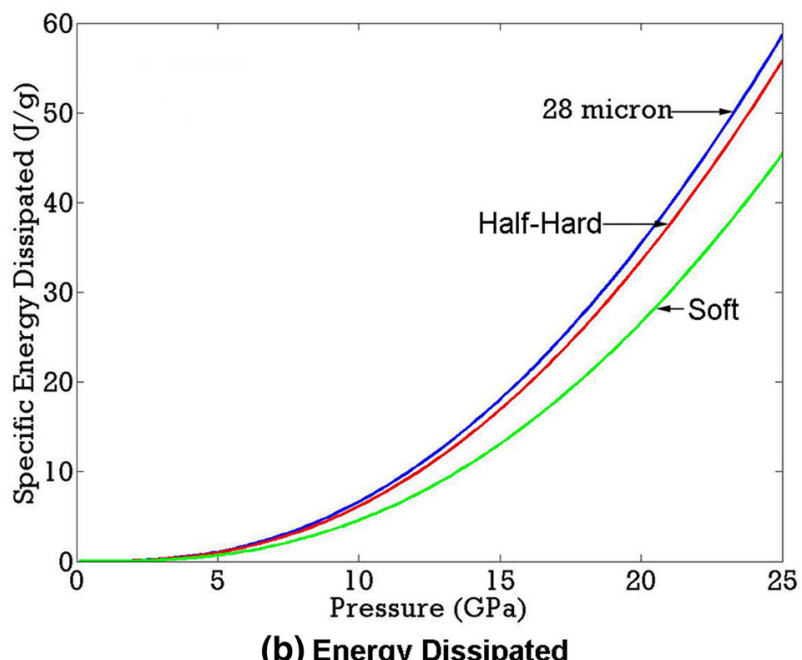

Fig. 9 The effect of material strength on the a dispersion and b dissipation of the shock front. Work hardening had a minimal effect on dispersion and a non-linear effect on dissipation

dispersion seen in the "uniform" configuration. While the increase in dissipation lowered the slope and increased the sound speed, the increase in dispersion acted oppositely. The end result was the slight upward shifting of the "uniform" EOS curve to higher shock wave speeds.

\section{Effect of Constituent Material Strength on Dispersion and Dissipation}

The shock fronts for the three material strengths investigated are presented in Fig. 9a. Once again, the curves are shifted along the abscissa. Material strength does not affect the geometry of the system, so no significant variations were expected or seen in the shock fronts of each configuration. There was some increase in dispersion between the "half-hard" and "soft" configurations. This represented 


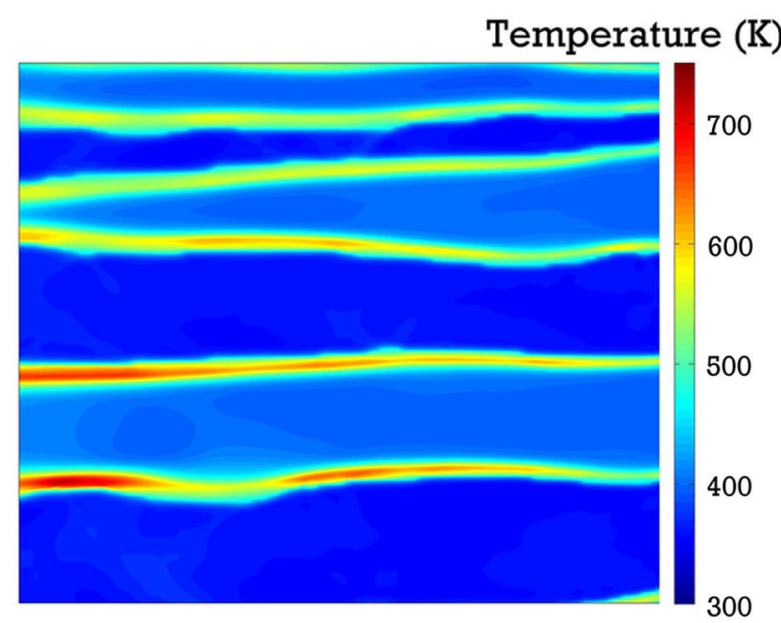

(a)

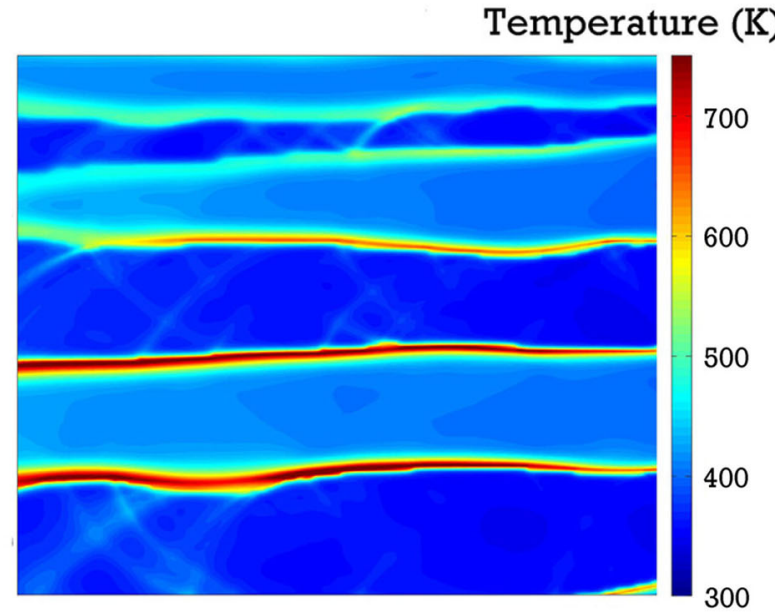

(b)

Fig. 10 High resolution AMR simulations showing the profiles for the a "soft" and b "half-hard" configurations. The increased compressibility of the "soft" configuration led to more deformation in the material layers, lowering interfacial strain compared to the "half-hard" configuration

differing areal changes of the layers during compression, which alter the geometry of the system slightly.

The dissipative responses for each yield strength are shown in Fig. 9b. The results revealed a non-linear trend of decreasing dissipation with decreasing material strength. The decrease in dissipation for a softer composite stemmed from the increased compressibility of the materials. With less work hardening, the interiors of each layer accommodated more deformation, isolating less strain at the interfaces and decreasing the bulk dissipation.

This behavior was seen in the high resolution temperature profiles for the "soft" and "half-hard" configurations presented in Fig. 10. The higher compressibility of the layers in the "soft" configuration reduced the interfacial

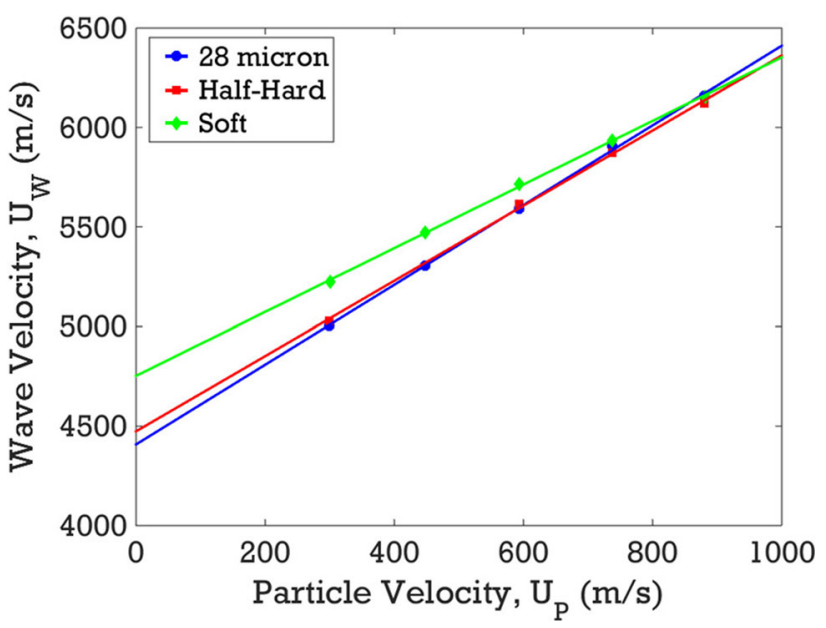

Fig. $11 U_{W}$ versus $U_{P}$ relationship for the "soft", "half-hard", and 28 micron composites for $U_{P}<1000 \mathrm{~m} / \mathrm{s}$. The markers correspond to the simulation results, while the lines represent the least squares fits

strains and temperatures generated in the composite. However, the decrease in dissipation was not directly proportional to the material strength. This observation was evident in the similar interfacial temperatures achieved in the "half-hard" and 28 micron configurations (Figs. 10b, $4 \mathrm{~b})$. The increased work hardening of the 28 micron configuration did not produce a large increase in the dissipation, since most of the deformation was already isolated at the interfaces.

The dissipative characteristics were also observed in the EOS response for each configuration, as shown in Fig. 11. The differing material strengths had essentially identical dispersive characteristics, due to their similar geometry. This meant the variations in their EOS responses were solely the result of their differing levels of dissipation. As stated previously, decreases in dissipation lower the $U_{W}$ versus $U_{P}$ slope and increase the sound speed, which was the observed result.

\section{Conclusions}

The effects of both interfacial and material properties on the dispersion and dissipation of shock waves traveling parallel to the interfaces in a laminar $\mathrm{Ni} / \mathrm{Al}$ composite were examined. Optimal bilayer spacings for both the dispersion and dissipation of a shock wave were identified. Both of these results were influenced by the number and nature of wave interactions in the composite, defining the equilibration time. As the equilibration time increased, the amount of energy dissipated at each interface increased. The increased energy dissipation at the interfaces was initially enough to offset the lose of interfacial area, causing a net increase in dissipation. Eventually, the 
increase in energy dissipated at each interface could not offset the loss of interfacial area and the total dissipation decreases. Interfacial structure altered the wave interactions and affected the dispersion of the system. With more interfacial area aligned with the propagating shock wave, there was increased interfacial strain and energy dissipated. Interfacial strength dramatically affected both dispersion and dissipation. When the materials moved freely, interfacial strain was eliminated as a dissipative mechanism, leaving only compression. The yield strength of the constituents did not strongly influence dispersion but did effect the dissipative response. With softer layers, the interiors accommodated more deformation, lowering the interfacial strain, and decreasing the dissipation of the system.

Acknowledgements Special Thanks to Adam Stover for fabricating the multilayer samples. This work was funded through MURI Grant No. N00014-07-1-0740, Dr. Cliff Bedford program manager, and involved the University of California at San Diego (Lead), the Johns Hopkins University, and the Georgia Institute of Technology. Sandia National Laboratories is a multi-program laboratory managed and operated by Sandia Corporation, a wholly owned subsidiary of Lockheed Martin Corporation, for the U.S. Department of Energys National Nuclear Security Administration under contract DE-AC0494AL85000.

\section{References}

1. Budiman A, Han S, Li N, Wei Q, Dickerson P, Tamura N, Kunz $\mathrm{M}$, Misra A (2012) Plasticity in the nanoscale $\mathrm{Cu} / \mathrm{Nb}$ singlecrystal multilayers as revealed by synchrotron Laue x-ray microdiffraction. J Mater Res 27(3):599-611

2. Kim Y, Budiman A, Baldwin J, Mara N, Misra A, Han S (2012) Microcompression study of Al-Nb nanoscale multilayers. J Mater Res 27(3):592-598

3. Lundergan C, Drumheller D (1971) Propagation of stress waves in a laminated plate composite. J Appl Phys 42(2):669-675

4. Oved Y, Luttwak G, Rosenberg Z (1978) Shock wave propagation in layered composites. J Compos Mater 12:84-96

5. Zhuang S, Ravichandran G, Grady D (2003) An experimental investigation of shock wave propagation in periodically layered composites. J Mech Phys Solids 51:245-265

6. Barker L (1971) A model for stress wave propagation in composite materials. J Compos Mater 5:140-162

7. Curran D, Seaman L, Austin M (1974) The use of artificial viscosity to compute one-dimensional wave propagation in composite materials. J Compos Mater 8:142-159

8. Drumheller D, Sutherland H (1973) A lattice model for stress wave propagation in composite materials. J Appl Mech 40:149-154

9. Chen X, Chandra N, Rajendran A (2004) Analytical solution to the plate impact problem of layered heterogeneous material systems. Int J Solids Str 41:4635-4659
10. Chen X, Chandra N (2004) The effect of heterogeneity on plane wave propagation through layered composites. Compos Sci Technol 64:1477-1493

11. Sun C, Achenbach J, Herrmann G (1968) Time-harmonic waves in a stratified medium propagating in the direction of the layering. J Appl Mech 35:408-411

12. Sun C, Achenbach J, Herrmann G (1968) Continuum theory for a laminated medium. J Appl Mech 35(3):467-475

13. Achenbach J, Hermann G (1968) Dispersion of free harmonic waves in fiber-reinforced composites. AIAA J 6(10):1832-1836

14. Peck J, Gurtman G (1969) Dispersive pulse propagation parallel to the interfaces of a laminated composite. J Appl Mech 36(3):479-484

15. Tsou F, Chou P (1969) Analytical study of Hugoniot in unidirectional fiber reinforced composites. J Compos Mater 3:500-514

16. Tsou F, Chou P (1970) The control-volume approach to hugoniot of macroscopically homogeneous composites. J Compos Mater 4:526-537

17. Torvik P (1970) Shock propagation in a composite material. J Compos Mater 4:296-309

18. Zhao S, Germann T, Strachan A (2007) Molecular dynamics simulation of dynamical response of perfect and porous $\mathrm{Ni} / \mathrm{Al}$ nanolaminates under shock loading. Phys Rev B 76:1

19. Cherukara M, Weihs T, Strachan A (2015) Molecular dynamics simulations of the reaction mechanism in $\mathrm{Ni} / \mathrm{Al}$ reactive intermetallics. Acta Mater 96:1-9

20. Cherukara M, Germann T, Kober E, Strachan A (2014) Shock loading of granular Ni/Al composites. Part 1: mechanics of loading. J Phys Chem C 45:26377-26386

21. Specht $P$, Thadhani N, Weihs T (2012) Configurational effects on shock wave propagation in Ni-Al multilayer composites. J Appl Phys 111:073527

22. Xun Y, Lunking D, Besnoin E, Van Heerden D, Weihs T, Knio O (2011) U.S. Patent Application 20110027547

23. Wei C, Maddox B, Stover A, Weihs T, Nesterenko V, Meyers M (2011) Reaction in Ni-Al laminates by laser-shock compression and spalling. Acta Mater 59:5276-5287

24. Spowart J, Maruyama B, Miracle D (2001) Multi-scale characterization of spatially heterogeneous systems: implications for discontinuously reinforced metal-matrix composite microstructures. Mater Sci Eng A 307:51-66

25. Sandia National Laboratories, CTH v8.1, v9.1, and v10.2 (20072011)

26. Specht PE (2013) Shock compression response of aluminumbased intermetallic-forming reactive systems. Ph.D. thesis, Georgia Institute of Technology

27. Specht P, Thadhani N, Weihs T (2013) Shock compression response of cold-rolled Ni/Al multilayer composites. In: MRS Proceedings, vol 1521. Cambridge University Press

28. Johnson G, Cook W (1983). In: Proceedings of the Seventh International Symposium on Ballistics, The Hague, The Netherlands, pp 541-547

29. Steinberg D, Cochran S, Guinan M (1980) A constitutive model for metals applicable at high-strain rate. J Appl Phys 51(3):1498-1504 